\title{
A Systematic Literature Review for Evaluating the Clinical Usefulness of the Customized Vestibular Exercise
}

\author{
Seung Hee Lee ${ }^{1}$, Keun young Park ${ }^{1}$, Byungkun Kim², Gyu Cheol Han ${ }^{3}$, Sung Won Chae \\ Gi Jung $\mathrm{Im}^{4}$, Jong Seok Bae ${ }^{5}$, Young Hwangbo ${ }^{6}$, Kyurin Hwang ${ }^{7}$, and Byung Don Lee \\ ${ }^{I}$ Division for New Health Technology Assessment, National Evidence-Based Healthcare Collaborating Agency, Seoul; and \\ ${ }^{2}$ Deparment of Neurology, Eulji University College of Medicine, Seoul; and ${ }^{3}$ Department of Otorhinolaryngology-Head and \\ Neck Surgery, Gachon University of Medicine and Science, Graduate School of Medicine, Seongnam; and \\ ${ }^{4}$ Department of Otorhinolaryngology-Head and Neck Surgery, Korea University College of Medicine, Seoul; and \\ ${ }^{5}$ Department of Neurology, Kangdong Sacred Heart Hospital, Hallym University College of Medicine, Anyang; and \\ ${ }^{6}$ Department of Preventive Medicine, Soonchunhyang University College of Medicine, Cheonan; and \\ ${ }^{7}$ Department of Otorhinolaryngology-Head and Neck Surgery, Soonchunhyang University College of Medicine, Seoul, Korea
}

\section{맞춤전정운동의 임상적 유용성 평가를 위한 체계적 문헌고찰}

\author{
이승희 ${ }^{1} \cdot$ 박근영 $^{1} \cdot$ 김병건 $^{2} \cdot$ 한규철 $^{3} \cdot$ 채성원 $^{4} \cdot$ 임기정 $^{4} \cdot$ 배종석 $^{5} \cdot$ 황보영 $^{6} \cdot$ 황규린 $^{7} \cdot$ 이병돈 $^{7}$ \\ 한국보건의료연구원 신의료기술평가사업본부, ${ }^{1}$ 을지대학교 의과대학 신경과학교실, ${ }^{2}$ 가천대학교 의과대학 이비인후과학교실, ${ }^{3}$ \\ 고려대학교 의과대학 이비인후과학교실, ${ }^{4}$ 한림대학교 의과대학 강동성심병원 신경과학교실, \\ 순천향대학교 의과대학 예방의학교실, ${ }^{6}$ 순천향대학교 의과대학 이비인후과학교실 ${ }^{7}$
}

\author{
Received March 7, 2018 \\ Revised May 7, 2018 \\ Accepted May 14, 2018 \\ Address for correspondence \\ Byung Don Lee, MD, PhD \\ Department of Otorhinolaryngology- \\ Head and Neck Surgery, \\ Soonchunhyang University \\ College of Medicine, \\ 59 Daesagwan-ro, Yongsan-gu, \\ Seoul 04401, Korea \\ Tel +82-2-709-9360 \\ Fax $+82-2-709-9362$ \\ E-mail bdlee12@schmc.ac.kr
}

Background and Objectives This study aims to evaluate the usefulness of customized vestibular exercise through literature review.

Materials and Method We searched several literature database such as Ovid-MEDLINE, Ovid-EMBASE, and Cochrane Library etc., with the following inclusion criteria: 1) studies of patients with dizziness and balance disorders, 2) studies in which a customized vestibular exercise was performed, and 3) studies in which one or more of the appropriate medical outcomes have been reported. At the same time, we excluded the following: 1) non-human studies and pre-clinical studies, 2) non-original articles, for example, non-systematic reviews, editorial, letter and opinion pieces, 3) research not published in Korean and English, 4) grey literature (thesis, congress or conference materials, abstract etc.), and 5) case studies. Finally, 10 studies were selected and analyzed.

Results The safety of customized vestibular exercise was reported in three documents which reported no side effects related to the procedure. The effectiveness of customized vestibular exercise was proven by the assessment of symptom change, functional change, and other physiological measures based on a total of 10 randomized clinical trial studies.

Conclusion For patients with vestibular dysfunction, a customized vestibular exercise can be a safe and effective technique for improving dizziness and balance function.

Korean J Otorhinolaryngol-Head Neck Surg 2018;61(7):325-32

Key Words Customized vestibular exercise · Dizziness · Vestibular disorder.

This is an Open Access article distributed under the terms of the Creative Commons Attribution Non-Commercial License (https:/creativecommons.org/licenses/by-nc/4.0) which permits unrestricted non-commercial use, distribution, and reproduction in any medium, provided the original work is properly cited. 


\section{서 론 하고자 하였다.}

전정계는 인체의 평형을 유지하는 역할을 하며 말초전정계 와 중추전정계로 구성된다. 말초전정계는 공간 속에서 머리 의 움직임을 감지하여 전정핵과 소뇌로 전달하며, 중추전정 계는 말초전정계의 정보를 시각, 체성감각, 자율신경계의 정 보와 통합하여 머리의 위치와 움직임, 중력의 방향을 확인하 여 시선을 고정하고 안정된 머리와 몸의 자세를 유지하는 역 할을 한다. 보상되지 않은 전정기능 저하는 자세 불안정, 머리 움직임시 시야 흐림, 불균형을 동반한 어지러움 주관적 호소 를 야기시킨다. ${ }^{1)}$

급성 전정기능 소실 후의 증상 회복은 중추보상에 의해 이 루어진다. 급성기 증상이 어느정도 가라앉은 후에는 조기에 전정재활치료라고 부르는 재활 치료를 시작해야 한다. 전정재 활은 말초성 전정장애 환자에서 널리 사용되고 있으면) 급성 편측성 전정기능 소실 환자에서 특히 치료의 유효성이 널리 인정받고 있다. 전정재활의 목적은 시각-전정 상호작용과 움 직임에 대한 저항력을 향상시키기 위한 눈과 머리의 움직임을 포함하여 전정 보상 과정을 자극하는 데 있다.3) 이를 통해 사 물의 상을 정확히 인지하게 함으로써 시야가 안정되고 전체적 인 신체 평형감을 회복하여 보행 시 균형을 잃지 않게 하려는 것이다. 이는 환자의 주관적인 불균형 및 넘어짐에 대한 위험 감소로 이어지고 결국 전정장애로 인한 사회적 고립을 방지하 게 된다. 지금까지의 전정재활치료 방법은 의료기관의 현실적 인 문제들(시간, 설비, 인력, 치료 수가의 미책정 등)로 인하여 대부분 외래에서 운동방법에 대한 교육을 받고 환자 스스로 가정에서 치료하는 가정기반치료(home base training)가 중 심이 되었다. 하지만 이러한 가정기반치료의 경우 치료순응 도가 일정하지 않아 치료 효과가 제한적인 단점이 있어왔다.

맞춤전정운동(customized vestibular exercise)은 이러한 가정기반치료의 한계점을 극복하고자 어지럼 및 균형장애 환자를 대상으로 어지럼 및 균형장애 개선을 목적으로 환자 의 증상과 장애에 맞추어 주시 안정 및 평형 유지에 필요한 개별화된 운동을 의료기관에서 직접 처방, 교육, 훈련시키는 기술이다. 환자의 특별한 요구에 맞추어 운동프로그램을 채 택할 수 있고 의료기관에서 직접 재활훈련과정에 개입할 수 있어 보다 적극적인 치료순응도의 확보가 가능하다. 이러한 많 은 장점으로 인해 최근 전정기능장애 환자 치료의 일차 치료 양식이 되었으며, ${ }^{4}$ 우리나라에서도 임상적 유용성을 인정하 여 2016년 7월 4일 대한평형의학회에 의해서 신의료기술평 가신청을 하기에 이르렀다.

따라서 본 연구에서는 맞춤전정운동에 대한 여러 가지 문 헌들을 분석하여 맞춤전정운동의 안전성 및 유효성을 평가

\section{재료 및 방법}

\section{연구 설계}

본 연구는 체계적 문헌고찰 방법을 통하여 맞춤전정운동 의 안전성 및 유효성을 확인하였다. 연구목적에 따른 문헌검색 전략은 patient-intervention-comparators-outcome(PICO) 을 통해 구체화하고 이를 바탕으로 검색어를 선정하여 문헌 검색 및 최종 문헌선택 과정을 수행하였다.

\section{문헌검색}

\section{PICO}

본 연구의 대상 환자(P)는 임상 전문가와의 논의를 통해 어 지럼과 균형장애 환자로 명확히 하였으며 중재시술(I)은 맞춤 전정운동이었다. 비교시술 $(\mathrm{C})$ 은 기성식 전정재활 치료, 무치 료, 약물치료로 보았으며, 의료결과(O)로 안전성 지표는 시술 관련 부작용, 유효성 지표는 기능변화[dizziness handicap inventory(DHI), dynamic gait index(DGI), Timed up and go test], 증상변화[visual analog scale(VAS), vertigo symptom scale(VSS)], 기타 생리적 측정(자세 안정성 검사, 동적 시력) 으로 평가하였다.

\section{문헌검색 및 최종 문헌선택 과정}

문헌검색은 국내와 국외 데이터베이스로 나누어 각각 2016 년 10월 31일, 2016년 10월 13일에 검색을 수행하였다. 국내 데 이터베이스는 KoreaMed, 국립중앙도서관, 국회도서관, 국가 자료공동목록시스템, 한국교육학술정보원, 학술데이터베이 스검색, 과학기술학회마을, 한국의학논문데이터베이스를 이 용하였고, 국외 데이터베이스는 Medline, Embase, Cochrane Library 및 Trip database를 이용하였다. 검색어는 각 데이터 베이스별 특성을 고려하여 대상 환자 및 중재시술에 초점을 맞추어 검색하였으며, 검색과정에서 언어 제한은 이루어지지 않았다. 검색전략에 따라[(exp rehabilitation/OR rehabilitation.mp. OR exp exercise/OR exercise.mp. OR exp therapeutics/OR therapy.mp. OR exp physical therapy modalities/ OR physiotherapy.mp. OR program*.mp.) AND (individualized.mp. OR customized.mp. OR personalized.mp. OR supervised.mp.) AND (assess*.mp. OR estimate*.mp. evaluate*.mp.)] 관련 질환의 검색어를 활용하여 수기 검색을 포 함하여 총 920편의 문헌을 검색하였다.

문헌선택은 선택 및 배제 기준에 근거하여 2 명의 평가자가 
독립적으로 실시하였다. 초록을 중심으로 일차적으로 선택 및 배제를 실시하고, 평가자 간 문헌선택에 이견이 있는 경우 전문을 확인하여 선택 및 배제를 최종적으로 시행하였다. 문 헌선택기준은 어지럼과 균형장애 환자를 대상으로 한 연구,

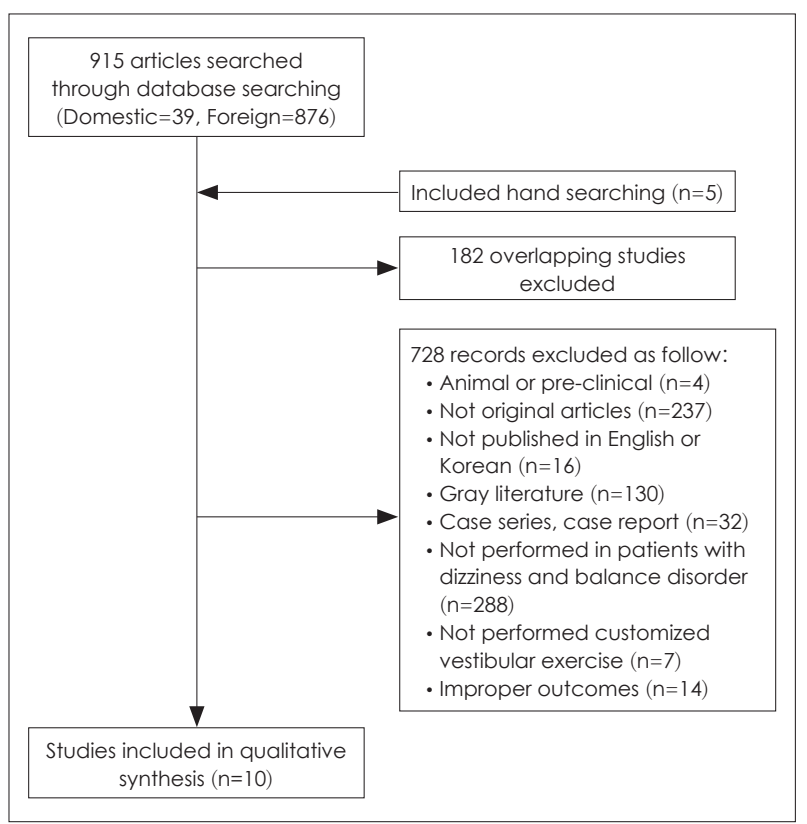

맞춤전정운동이 시행된 연구, 적절한 의료결과가 하나 이상 보고된 연구로 하였으며 배제 기준은 동물실험 또는 전임상 시험 연구, 원저가 아닌 연구, 한국어 및 영어로 출판되지 않 은 연구, 회색 문헌, 증례연구를 기준으로 하였다. 중복 검색된 문헌 182편을 제외한 738편을 토대로 선택 및 배제 기준을 적용하여 총 10 편의 무작위 임상시험연구가 최종평가에 포 함되었다(Fig. 1).

\section{문헌의 질 평가}

선택된 문헌의 질 평가는 영국 Scottish Intercollegiate Guidelines Network(SIGN)의 방법론 체크리스트(methodology checklist, 2004)를 이용하였으며 2명의 평가자가 독립적으 로 수행하였다. $\mathrm{SIGN}$ 의 질 평가 도구는 연구유형에 따라 필 수항목을 선정하고, 질 평가항목에 거의 모든 또는 모든 기 준이 충족되는 경우 ‘++', 몇 가지 기준이 충족된 경우 ‘+', 거 의 모든 또는 모든 기준이 충족되지 않는 경우 '-'로 평가하 였으며, 질 평가 결과에 따른 근거 수준은 Table 1과 같다. 평 가자 간 질 평가 결과에 이견은 없었으며, 관련 문헌의 질 평 가 결과는 1'++' 2편, 1‘+' 8편이었다. 또한 질 평가 결과를 반 영한 근거의 수준에 따른 권고등급은 Table 2와 같다.

Fig. 1. Flow chart of literature selection process.

Table 1. Scottish Intercollegiate Guidelines Network criteria for assignment of levels of evidence

\begin{tabular}{|c|c|}
\hline Level & Description \\
\hline $1++$ & High quality meta-analyses, systematic reviews of RCTs, or RCT with a very low risk of bias \\
\hline $1+$ & Well-conducted meta-analyses, systematic reviews, or RCTs with a low risk of bias \\
\hline $1-$ & Meta-analyses, systematic reviews, or RCTs with a high risk of bias \\
\hline $2++$ & $\begin{array}{l}\text { High quality systematic reviews of case control or cohort or studies } \\
\text { High-quality case-control or cohort studies with a very low risk of confounding or bias and a high probability that the } \\
\text { relationship is causal }\end{array}$ \\
\hline $2+$ & $\begin{array}{l}\text { Well-conducted case-control or cohort studies with a low risk of confounding or bias and a moderate probability that } \\
\text { the relationship is causal }\end{array}$ \\
\hline $2-$ & Case-control or cohort studies with a high risk of confounding or bias and a significant risk that the relationship is not causal \\
\hline 3 & Non-analytic studies, e.g., case reports, case series \\
\hline 4 & Expert opinion \\
\hline
\end{tabular}

Table 2. Scottish Intercollegiate Guidelines Network criteria for assignment of levels of grades of recommendation

\begin{tabular}{ll}
\hline Level & Description \\
\hline A At least one meta-analysis, systematic review, or RCT rated as $1++$, and directly applicable to the target population; \\
or a body of evidence consisting principally of studies rated as $1+$, directly applicable to the target population, and \\
demonstrating overall consistency of results
\end{tabular}

RCT: randomized controlled trial 


\section{자료 추출}

자료 추출은 기본 서식을 바탕으로 임상 전문가의 의견을 반영하여 서식을 확정받았으며, 문헌에 기술된 공통된 내용 과 각 문헌별로 결과에 영향을 주는 연구대상의 특성 및 안 전성 및 유효성에 대한 자료를 추출하였다. 빠짐없이 추출하 기 위하여 3차례 반복하여 수행한 뒤 2차례 7명의 임상 전문 가 회의를 통해 최종 확인되었다.

\section{결 과}

\section{대상 문헌의 일반 특성}

맞춤전정운동의 안전성 및 유효성 평가에 선택된 문헌은 무작위 임상시험연구 총 10 편으로 모두 국외 문헌이었다. 연 구국가는 이탈리아, 터키, 벨기에, 미국 등으로 다양하였으며 무치료 또는 기성식 전정재활과 비교한 연구였다(Table 3). 연구 대상은 전정장애 8 편, 청각 신경종 절제 1 편, 어지럼증 1편이었다.

\section{동 기술의 안전성}

맞춤전정운동의 안전성은 3편의 문헌(Marioni 등, ${ }^{2)}$ Marioni 등, ${ }^{5}$ Herdman 등 $)^{6}$ )에서 보고하였으며, 동 시술 관련 어떤 부
작용도 없었다고 보고하였다.

\section{동 기술의 유효성}

맞춤전정운동의 유효성은 총 10 편의 무작위 임상시험연구 (기성식 전정재활 치료와 비교 2편, 무치료 군과 비교 8편)를 근거로 증상 변화, 기능 변화, 기타 생리적 측정을 지표로 평가 하였다.

첫째, 기성식 전정재활 치료와의 비교연구(2편)의 연구 대상 은 모두 전정장애 환자였다. 증상(현기증, 균형) 변화, 기능 변 화(어지럼증 자가진단검사, DHI), 기타 생리적 측정(자세 안정 성 검사)은 군간 유의한 차이가 없었다(Table 4).

둘째, 무치료 군과 비교한 연구(8편)의 연구대상은 전정장 애(7편)와 만성 어지럼증(1편)이었다. 전정장애 환자 대상의 문헌(7편)에서 증상 변화는 현훈(1편), 불안정성(1편), 불안(1 편)이 모두 중재 군이 대조군보다 유의하게 향상되었다고 보고 하였다. 기능 변화는 일차 유효성 지표인 DHI(2편) 점수가 중 재 군이 대조군보다 유의하게 향상되었으며 DGI(2편), Timed up and go test(1편) 점수는 군간 유의한 차이가 없었다. 기타 생리적 측정으로 시행된 자세 안정성 검사와 동적 시력 검사 결과는 2편에서 이질적으로 제시하였다(Table 5). 만성 어지럼 증 대상의 문헌(1편)에서 증상 변화(현훈), 기능 변화(DHI), 기

Table 3. Study characteristics of included studies

\begin{tabular}{|c|c|c|c|c|c|}
\hline $\begin{array}{l}\text { Study } \\
\text { design }\end{array}$ & Author (year) & Indication (n) & Intervention & Comparator & $\begin{array}{l}\text { Level of } \\
\text { evidence }\end{array}$ \\
\hline \multirow[t]{2}{*}{$\overline{\mathrm{RCT}}$} & Marioni, et al. $(2013)^{2)}$ & $\begin{array}{l}\text { Unilateral peripheral } \\
\text { vestibular disorder (30) }\end{array}$ & $\begin{array}{l}\text { Customized vestibular } \\
\text { exercise }\end{array}$ & No treatment & $1+$ \\
\hline & & $\begin{array}{l}\text { Unilateral peripheral } \\
\text { vestibulopathy (28) }\end{array}$ & $\begin{array}{l}\text { Customized vestibular } \\
\text { exercise+Traditional } \\
\text { vestibular exercise } \\
\text { (home) }\end{array}$ & $\begin{array}{l}\text { Traditional vestibular } \\
\text { exercise (home) }\end{array}$ & $1+$ \\
\hline RCT & Giray, et al. $(2009)^{4)}$ & $\begin{array}{l}\text { Unilateral Vestibular } \\
\text { disorder (42) }\end{array}$ & $\begin{array}{l}\text { Customized vestibular } \\
\text { exercise }\end{array}$ & No treatment & $1+$ \\
\hline RCT & Teggi, et al. (2009)9) & $\begin{array}{l}\text { Acute vestibular } \\
\text { disorder (40) }\end{array}$ & $\begin{array}{l}\text { Customized vestibular } \\
\text { exercise }\end{array}$ & No treatment & $1+$ \\
\hline RCT & Vereeck, et al. $(2008)^{10)}$ & $\begin{array}{l}\text { Acoustic neuroma } \\
\text { resection (53) }\end{array}$ & $\begin{array}{l}\text { Customized vestibular } \\
\text { exercise }\end{array}$ & No treatment & $1++$ \\
\hline RCT & Herdman, et al. $(2007)^{6)}$ & $\begin{array}{l}\text { Bilateral vestibular } \\
\text { hypofunction (13) }\end{array}$ & $\begin{array}{l}\text { Customized vestibular } \\
\text { exercise }\end{array}$ & No treatment & $1++$ \\
\hline RCT & Kammerlind, et al. $(2005)^{3)}$ & $\begin{array}{l}\text { Unilateral vestibular } \\
\text { loss (54) }\end{array}$ & $\begin{array}{l}\text { Customized vestibular } \\
\text { exercise+Traditional } \\
\text { vestibular exercise } \\
\text { (home) }\end{array}$ & $\begin{array}{l}\text { Traditional vestibular } \\
\text { exercise (home) }\end{array}$ & $1+$ \\
\hline RCT & Yadley, et al. $(2004)^{8)}$ & Chronic dizziness (170) & $\begin{array}{l}\text { Customized vestibular } \\
\text { exercise }\end{array}$ & No treatment & $1+$ \\
\hline RCT & Herdman, et al. (2003) & $\begin{array}{l}\text { Unilateral vestibular } \\
\text { hypofunction (21) }\end{array}$ & $\begin{array}{l}\text { Customized vestibular } \\
\text { exercise }\end{array}$ & No treatment & $1+$ \\
\hline RCT & Yardley, et al. $(1998)^{7)}$ & Dizziness or vertigo (143) & $\begin{array}{l}\text { Customized vestibular } \\
\text { exercise }\end{array}$ & No treatment & $1+$ \\
\hline
\end{tabular}

RCT: randomized controlled trial 
Table 4. Summary of results (customized vestibular exercise vs. traditional vestibular exercise)

\begin{tabular}{|c|c|c|c|c|c|c|c|}
\hline Author (year) & $\begin{array}{l}\text { No. of patients } \\
\text { (intervention/ } \\
\text { comparators) }\end{array}$ & Variables & \multicolumn{2}{|c|}{ Intervention } & \multicolumn{2}{|c|}{ Comparators } & p-value \\
\hline \multicolumn{2}{|c|}{ Symptomatic changes } & & \multicolumn{2}{|c|}{ Before } & \multicolumn{2}{|c|}{ After 6 month } & $\mathrm{p}$ (intergroup) \\
\hline \multirow[t]{3}{*}{ Kammerlind, et al. $(2005)^{3)}$} & \multirow[t]{3}{*}{$54(28 / 26)$} & $\begin{array}{l}\text { Vertigo during } \\
\text { movement }\end{array}$ & \multicolumn{2}{|c|}{$8.6 \pm 12.2$} & \multicolumn{2}{|c|}{$13.9 \pm 20.5$} & 0.977 \\
\hline & & Vertigo at rest & \multicolumn{2}{|c|}{$8.0 \pm 14.5$} & \multicolumn{2}{|c|}{$3.2 \pm 7.6$} & 0.138 \\
\hline & & Balance problems & \multicolumn{2}{|c|}{$14.2 \pm 20.0$} & \multicolumn{2}{|c|}{$9.8 \pm 14.9$} & 0.357 \\
\hline \multicolumn{2}{|c|}{ Functional changes (DHI) } & & Before & After 6 week & Before & After 6 week & $p$ (intergroup) \\
\hline \multirow[t]{4}{*}{ Marioni, et al. $(2013)^{2)}$} & $28(14 / 14)$ & Functional & $13.4 \pm 8.4$ & $9.4 \pm 7.2$ & $12.4 \pm 8.3$ & $10.0 \pm 8.3$ & 0.90 \\
\hline & & Emotional & $11.1 \pm 9.3$ & $8.4 \pm 10.0$ & $11.4 \pm 7.4$ & $8.7 \pm 7.5$ & 0.62 \\
\hline & & Physical & $11.1 \pm 6.2$ & $8.9 \pm 7.7$ & $9.3 \pm 4.9$ & $6.7 \pm 3.6$ & 0.61 \\
\hline & & Total score & $35.7 \pm 20.7$ & $26.7 \pm 22.7$ & $33.1 \pm 17.2$ & $25.4 \pm 17.2$ & 0.90 \\
\hline \multicolumn{2}{|c|}{ Computerized posturography } & & Before & After 6 week & Before & After 6 week & $\mathrm{p}$ (intergroup) \\
\hline \multirow[t]{4}{*}{ Marioni, et al. $(2013)^{2)}$} & $28(14 / 14)$ & FIRM EO & $0.3 \pm 0.3$ & $0.2 \pm 0.2$ & $0.2 \pm 0.3$ & $0.2 \pm 0.2$ & $\mathrm{~ns}$ \\
\hline & & FIRM EC & $0.5 \pm 0.5$ & $0.3 \pm 0.3$ & $0.4 \pm 0.5$ & $0.3 \pm 0.3$ & ns \\
\hline & & FOAM EO & $1.1 \pm 0.6$ & $1.0 \pm 0.6$ & $0.9 \pm 0.6$ & $0.9 \pm 0.6$ & ns \\
\hline & & FOAM EC & $3.1 \pm 1.3$ & $2.9 \pm 1.1$ & $2.4 \pm 0.6$ & $2.2 \pm 0.7$ & ns \\
\hline
\end{tabular}

DHI: dizziness handicap inventory, FIRM EC: standing on a firm surface with eyes closed, FIRM EO: standing on a firm surface with eyes open, FOAM EC: standing on a foam surface with eyes closed, FOAM EO: standing on a foam surface with eyes open, ns: not significant

타 생리적 측정(자세 안정성검사)이 모두 중재 군이 대조군보 다 유의한 향상이 있었다고 보고하였다(Table 6).

\section{고 찰}

맞춤전정운동은 어지럼 및 균형장애 환자를 대상으로 어 지럼 및 균형장애 개선을 목적으로 환자의 증상과 장애에 맞 추어 주시 안정 및 평형 유지에 필요한 개별화된 운동을 처 방, 교육, 훈련시키는 기술로, 동 기술의 안전성 및 유효성에 대 해 문헌 선택의 기준을 정하여 10편의 무작위 임상시험연구 (기성식 전정재활 치료와 비교 2편, 무치료 군과 비교 8편) 논문을 선택하여 평가하였다. 10편의 문헌에서 맞춤형 전정 재활 운동이 어지럼증 환자의 증상을 완화시키고, 자세 안정 성을 향상시켰으며 어지럼증과 연관된 여러 가지 장애들을 향상시킴을 알 수 있었다.

증상의 변화를 주로 연구한 논문으로는 4편의 논문이 있 었으며 기성식 재활치료와 비교한 논문이 1편(Kammerlind 등 $^{3)}$, 치료를 하지 않은 군과 비교한 논문이 4편(Giray 등, Yardeley 등,8) Teggi 등 $\left.{ }^{91}\right)$ 이었다. Kammerlind 등 ${ }^{3)}$ 의 무작위 임상시험연구는 급성 편측성 전정소실 환자 54명을 대상으 로 맞춤전정운동과 함께 가정식 운동프로그램(home exercise) 을 같이 시행한 경우와 가정 운동프로그램만을 시행한 경우 를 VAS 점수를 통해 비교하였다. 치료 후 운동 중 현기증, 휴 식 중 현기증, 균형문제를 측정하였으며 치료 6개월 후 VAS 는 군간 유의한 차이가 없어 맞춤전정운동이 기존의 기성식
전정재활 치료에 비해 유효한 증상의 개선이 있음을 알 수 있었다. 무치료 군과 비교한 연구 중 Yardeley 등묘의 2편의 연구는 VSS를 사용하였으며 Giray 등과 과 Teggi 등)의 연구 는 VAS를 사용하였다. 4편의 연구 모두 증상의 개선이 중재 군이 대조군보다 유의한 결과를 나타내었다.

어지럼증으로 인한 기능의 변화를 측정하기 위한 평가항목 으로는 DHI점수와 DGI 및 Timed up and go 검사를 사용 하였다. Marioni 등 ${ }^{5}$ 의 무작위 임상시험연구는 중추성 전정 기능장애 28 명을 대상으로 맞춤전정운동과 가정 운동프로 그램(home exercise)을 병행한 경우와, 기존의 가정 운동프 로그램 단독으로 시행한 경우를 6주간 관찰하였을 때, 두 군 간에 $\mathrm{DHI}$ 점수의 유의한 차이는 없었으나 맞춤전정운동을 시행한 군의 경우 기능, 감정 그리고 총점에서 치료 전후의 점 수에 유의한 차이를 보였고, 기존의 치료를 적용한 군은 치 료 전후에 단지 감정 척도에서만 유의한 향상을 보여 맞춤전 정운동이 좀 더 유효함을 어느 정도는 시사한다고 할 수 있다. 또한, 치료를 시행하지 않은 군과 맞춤전정운동을 시행한 군 과 비교를 하였을 때 DHI 점수를 측정한 3편의 연구(Giray 등 ${ }^{4)}$, Yardley 등 ${ }^{8)}$, Teggi 등 $\left.{ }^{9}\right)$ 에서 중재 군이 대조군보다 유의 하게 향상되었다. DGI 점수는 2편(Teggi 등ㄹ), Vereeck 등 ${ }^{10)}$ ) 에서 시행하였고 Timed up and go 검사는 1편(Vereeck 등ํ) 의 연구에서 시행하였다. 이들 연구에서는 중재 군과 대조군 의 유의한 차이를 보이지는 않았다.

생리적 측정값의 변화는 4편의 연구에서 보고하였다. 자세 안정성검사 결과는 2 편의 문헌에서 보고하였으며 1편(Marioni 
Table 5. Summary of results in vestibular disorder (customized vestibular exercise vs. no treatment)

\begin{tabular}{|c|c|c|c|c|c|c|c|}
\hline Author (year) & $\begin{array}{l}\text { No. of patients } \\
\text { (intervention/ } \\
\text { comparators) }\end{array}$ & Variables & Interv & rention & Comp & arators & p-value \\
\hline Symptomatic & changes & & Before & After & Before & After & $p$ (intergroup) \\
\hline $\begin{array}{l}\text { Yardeley, et al. } \\
(1998)^{7)}\end{array}$ & $\begin{array}{c}143(67 / 76) \\
6 \text { week }\end{array}$ & VSS & $10.9 \pm 9.3$ & $7.9 \pm 6.4$ & $13.0 \pm 12.4$ & $12.9 \pm 11.8$ & 0.005 \\
\hline & 6 month & & & $7.7 \pm 8.2$ & & $12.5 \pm 11.0$ & \\
\hline Giray, et al. (2009) & $\begin{array}{c}42(20 / 22) \\
4 \text { week }\end{array}$ & $\begin{array}{l}\text { VAS } \\
\text { (unsteadyness) }\end{array}$ & 4.45 & 1.35 & 3.60 & 2.90 & 0.003 \\
\hline Teggi, et al. (2009)9) & $\begin{array}{c}40(20 / 20) \\
25 \text { day }\end{array}$ & VAS (anxiety) & $28.2 \pm 12.9$ & $6.5 \pm 8.7$ & $29.3 \pm 12.7$ & $19.7 \pm 9.9$ & $<0.001$ \\
\hline Functional ch & anges (DHI) & & Before & After & Before & After & $p$ (intergroup) \\
\hline Giray, et al. (2009) & $\begin{array}{c}42(20 / 22) \\
4 \text { week }\end{array}$ & $\mathrm{DHI}$ & 64.00 & 22.00 & 58.00 & 60.00 & 0.000 \\
\hline Teggi, et al. (2009)9) & $40(20 / 20)$ & $\mathrm{DHI}$ & $51.2 \pm 8.9$ & $18.6 \pm 11.7$ & $50.7 \pm 8.7$ & $29.4 \pm 12.8$ & 0.002 \\
\hline & 25 day & DGI & $16.8 \pm 1.3$ & $22.6 \pm 1.1$ & $17.1 \pm 1.3$ & $20.1 \pm 1.1$ & 0.095 \\
\hline Vereeck, et al. & $53(31 / 22)$ & DGI & - & - & - & - & 0.054 \\
\hline$(2008)^{1 C}$ & 1 year & Timed up and go & - & - & - & - & 0.055 \\
\hline Physiological m & easurements & & Before & After & Before & After & $\mathrm{p}$ (intergroup) \\
\hline Posturography & & & & & & & \\
\hline $\begin{array}{l}\text { Marioni, et al. } \\
(2013)^{2)}\end{array}$ & $\begin{array}{c}30(15 / 15) \\
6 \text { week }\end{array}$ & FIRM EO & $0.2 \pm 0.2$ & $0.3 \pm 0.1$ & $0.3 \pm 0.1$ & $0.3 \pm 0.2$ & ns \\
\hline & & FIRM EC & $0.4 \pm 0.2$ & $0.3 \pm 0.1$ & $0.4 \pm 0.2$ & $0.4 \pm 0.2$ & ns \\
\hline & & FOAM EO & $0.6 \pm 0.2$ & $0.4 \pm 0.1$ & $0.7 \pm 0.2$ & $0.6 \pm 0.2$ & 0.03 \\
\hline & & FOAM EC & $2.6 \pm 0.7$ & $1.4 \pm 0.2$ & $2.8 \pm 0.6$ & $2.3 \pm 0.4$ & 0.001 \\
\hline Postural stability & & & & & & & \\
\hline $\begin{array}{l}\text { Giray, et al. } \\
(2009)^{4)}\end{array}$ & $\begin{array}{c}42(20 / 22) \\
4 \text { week }\end{array}$ & FIRM EO (d/s) & 0.25 & 0.20 & 0.20 & 0.40 & 0.013 \\
\hline & & FIRM EC (d/s) & 0.30 & 0.25 & 0.30 & 0.40 & 0.036 \\
\hline & & FOAM EO (d/s) & 1.00 & 0.70 & 0.75 & 0.85 & 0.008 \\
\hline & & FOAM EC $(d / s)$ & 2.60 & 1.85 & 2.05 & 2.25 & 0.013 \\
\hline & & mCTSIB- $\dagger(d / s)$ & 1.10 & 0.85 & 0.80 & 1.10 & 0.004 \\
\hline $\begin{array}{l}\text { Herdman, et al. } \\
(2007)^{6)}\end{array}$ & $\begin{array}{l}14(8 / 5) \\
6 \text { week }\end{array}$ & $\begin{array}{l}\text { Dynamic visual } \\
\text { acuity }\end{array}$ & $0.312 \pm 0.089$ & $0.185 \pm 0.072$ & $0.466 \pm 0.158$ & $0.439 \pm 0.151$ & 0.002 \\
\hline $\begin{array}{l}\text { Herdman, et al. } \\
(2003)^{111}\end{array}$ & $\begin{array}{r}21(13 / 8) \\
4 \text { week }\end{array}$ & $\begin{array}{l}\text { Dynamic visual } \\
\text { acuity }\end{array}$ & - & - & - & - & - \\
\hline
\end{tabular}

DHI: dizziness handicap inventory, DGI: dynamic gait index, FIRM EC: standing on a firm surface with eyes closed, FIRM EO: standing on a firm surface with eyes open, FOAM EC: standing on a foam surface with eyes closed, FOAM EO: standing on a foam surface with eyes open, mCTSIB-t: total score of modified clinical test for sensory interaction of balance, ns: not significant, VAS: visual analogue scale, VSS: vertigo symptom scale

등)에서는 부분적으로 중재 군이 대조군보다 유의하게 보고 하였고, 다른 1편(Giray 등)에서는 중재 군이 대조군보다 유 의하게 향상되었다고 보고하였다. 동적 시력 검사 결과는 2 편의 문헌에서 보고하였으며 1편(Herdman 등ㅎ)에서는 중재 군이 대조군보다 유의하게 향상되었으며 다른 1편(Herdman 등 $\left.{ }^{11)}\right)$ 에서는 전후에는 두군 모두 유의하게 향상되었으며 군간 비교 결과는 제시되지 않았다. 만성 어지럼증 대상의 문헌 1 편(Yardley 등ㅇ)에서 자세 안정성 검사가 중재 군이 대조군 보다 유의하게 향상되었다고 보고하였다.
맞춤전정운동의 안전성은 3편의 연구(Marioni 등,5), Herd$\operatorname{man}$ 등 $\left.)^{6}\right)$ 에서 보고하였으며, 동 시술 관련 어떤 부작용도 없 었다고 보고하였다. 약물 주입이나 수술이 아닌 개별화된 운 동을 통하여 어지럼 및 균형장애를 개선시키는 기술로, 환자 에게 직접적인 위해는 없으므로 맞춤전정운동을 시행함에 있어 안전성에 문제가 없을 것으로 보인다.

맞춤전정운동이 기존의 전정재활 운동과 유효성이 유사하 고 무치료 군과의 비교에서 $\mathrm{DHI}$ 점수 및 증상 변화가 대조 군보다 치료 군이 유의하게 향상되는 결과를 보여 임상적으로 
Table 6. Summary of results in chronic dizziness (customized vestibular exercise vs. no treatment exercise)

\begin{tabular}{|c|c|c|c|c|c|c|c|}
\hline \multirow{2}{*}{$\begin{array}{r}\text { Author (year) } \\
\text { Symptoma }\end{array}$} & \multirow{2}{*}{$\begin{array}{l}\text { No. of patients } \\
\text { (intervention/ } \\
\text { comparators) } \\
\text { c changes }\end{array}$} & \multirow[t]{2}{*}{ Variables } & \multicolumn{2}{|c|}{ Intervention } & \multicolumn{2}{|c|}{ Comparators } & \multirow{2}{*}{$\begin{array}{c}p \text {-value } \\
p \text { (intergroup) }\end{array}$} \\
\hline & & & Before & After 3 month & After 6 month & After 3 month & \\
\hline \multirow{2}{*}{$\begin{array}{l}\text { Yardeley, et al. } \\
(2004)^{8)}\end{array}$} & $170(83 / 87)$ & VSS & $16.57 \pm 11.28$ & $9.88 \pm 0.76$ & $14.70 \pm 9.21$ & $13.3 \pm 0.74$ & 0.001 \\
\hline & & $\begin{array}{c}\text { Movement } \\
\text { provoked } \\
\text { dizziness }\end{array}$ & $27.28 \pm 5.72$ & $14.55 \pm 1.19$ & $26.56 \pm 7.64$ & $20.69 \pm 1.14$ & 0.001 \\
\hline \multicolumn{2}{|c|}{ Functional changes (DHI) } & & Before & After 3 month & After 6 month & After 3 month & $\mathrm{p}$ (intergroup) \\
\hline $\begin{array}{l}\text { Yardeley, et al. } \\
(2004)^{8)}\end{array}$ & $170(83 / 87)$ & $\mathrm{DHI}$ & $40.98 \pm 22.52$ & $31.09 \pm 1.52$ & $37.89 \pm 19.74$ & $35.88 \pm 1.48$ & 0.026 \\
\hline \multicolumn{2}{|c|}{ Postural stability } & & Before & After 6 week & After 6 month & After 3 month & $p$ (intergroup) \\
\hline \multirow{2}{*}{$\begin{array}{l}\text { Yardeley, et al. } \\
(2004)^{8)}\end{array}$} & $170(83 / 87)$ & Eyes open & $586.49 \pm 249.27$ & $528.71 \pm 19.68$ & $561.38 \pm 278.66$ & $593.71 \pm 18.98$ & 0.019 \\
\hline & & Eyes closed & $897.99 \pm 459.94$ & $731.95 \pm 32.05$ & $820.27 \pm 422.45$ & $854.25 \pm 30.48$ & 0.006 \\
\hline
\end{tabular}

DHI: dizziness handicap inventory, VSS: vertigo symptom scale

의미가 있을 것으로 생각된다. 다만 적용 대상에 대하여 평가 에 포함된 대부분의 문헌 대상이 전정장애 환자를 대상으로 하였으므로 맞춤전정운동의 적용은 전정기능장애 환자를 대상으로 하는 것이 적절할 것으로 생각된다.

본 연구는 문헌 선택을 함에 있어서 맞춤전정운동을 적용 한 무작위 임상시험연구만을 선택하였으며 이에 따라 문헌 수 가 적고, 연구별로 추적 기간이 상이하며, 평가 지표가 상이하 여 정량적 분석(quantitative analysis)을 수행하지 못한 점이 본 연구의 한계이다. 또한, 선택된 문헌 중 국내 연구가 없어 국내의 전정장애 환자에 대한 맞춤전정운동의 결과를 본 연 구에 반영하지 못하였다

그럼에도 불구하고 맞춤전정운동은 전정기능장애 환자를 대상으로 할 때 어지럼 및 균형을 개선시키는데 있어 안전하 고 유효한 기술로 판단을 내릴 수 있다. 국내에서도 맞춤전정 운동이 신의료기술로 인정받게 되었으므로 향후 국내 전정 장애 환자들을 대상으로 한 연구가 활발해지는 계기가 되어 앞에서 지적한 연구의 한계점들이 상당 부분 극복될 것으로 생각된다.

\section{REFERENCES}

1) Hall CD, Herdman SJ, Whitney SL, Cass SP, Clendaniel RA, Fife TD, et al. Vestibular rehabilitation for peripheral vestibular hypofunction: an evidence based clinical practice guideline: from the American physical therapy association neurology section. J Neurol Phys Ther 2016;40(2):124-55.
2) Marioni G, Fermo S, Zanon D, Broi N, Staffieri A. Early rehabilitation for unilateral peripheral vestibular disorders: a prospective, randomized investigation using computerized posturography. Eur Arch Otorhinolaryngol 2013;270(2):425-35.

3) Kammerlind AS, Ledin TE, Odkvist LM, Skargren EI. Effects of home training and additional physical therapy on recovery after acute unilateral vestibular loss--a randomized study. Clin Rehabil 2005; 19(1):54-62.

4) Giray M, Kirazli Y, Karapolat H, Celebisoy N, Bilgen C, Kirazli T. Short-term effects of vestibular rehabilitation in patients with chronic unilateral vestibular dysfunction: a randomized controlled study. Arch Phys Med Rehabil 2009;90(8):1325-31.

5) Marioni G, Fermo S, Lionello M, Fasanaro E, Giacomelli L, Zanon $\mathrm{S}$, et al. Vestibular rehabilitation in elderly patients with central vestibular dysfunction: a prospective, randomized pilot study. Age (Dordr) 2013;35(6):2315-27.

6) Herdman SJ, Hall CD, Schubert MC, Das VE, Tusa RJ. Recovery of dynamic visual acuity in bilateral vestibular hypofunction. Arch Otolaryngol Head Neck Surg 2007;133(4):383-9.

7) Yardley L, Beech S, Zander L, Evans T, Weinman J. A randomized controlled trial of exercise therapy for dizziness and vertigo in primary care. Br J Gen Pract 1998;48(429):1136-40.

8) Yardley L, Donovan-Hall M, Smith HE, Walsh BM, Mullee M, Bronstein AM. Effectiveness of primary care-based vestibular rehabilitation for chronic dizziness. Ann Intern Med 2004;141(8): 598-605.

9) Teggi R, Caldirola D, Fabiano B, Recanati P, Bussi M. Rehabilitation after acute vestibular disorders. J Laryngol Otol 2009;123(4):397-402.

10) Vereeck L, Wuyts FL, Truijen S, De Valck C, Van de Heyning PH. The effect of early customized vestibular rehabilitation on balance after acoustic neuroma resection. Clin Rehabil 2008;22(8):698-713.

11) Herdman SJ, Schubert MC, Das VE, Tusa RJ. Recovery of dynamic visual acuity in unilateral vestibular hypofunction. Arch Otolaryngol Head Neck Surg 2003;129(8):819-24. 


\section{정답 및 해설}

답 (5) 고실사구종(glomus tympanicum) 환자의 예로 catecholamine이나 neuropeptide인 serotonin 등을 분비하는 부종양 증후군(paraneoplastic syndrome)이 나타날 수 있어, 술 전에 검사로 확인하여 한다(이비인후과학: 일조각;2009. p.986, Cummings 6th edition; 2739).

해 설 (1) 일차적 치료는 수술적 제거이며, 재발하거나 완전 절제가 불가능한 경우 방사선치료를 고려해볼 수 있다.

(2) 안면신경초종의 경우 안면 마비가 흔하게 나타난다.

(3) 뇌 신경 마비는 주로 경정맥사구종 glomus jugulare에서 종종 나타난다.

(4) 고실사구종의 경우 크기가 작아 보통은 색전술이 필요하지 않다. 